\section{Cardiovascular Risk Reduction With Liraglutide: An Exploratory Mediation Analysis of the LEADER Trial}

Diabetes Care 2020;43:1546-1552 | https://doi.org/10.2337/dc19-2251
John B. Buse, ${ }^{1}$ Stephen C. Bain, ${ }^{2}$ Johannes F.E. Mann, ${ }^{3,4}$ Michael A. Nauck, ${ }^{5}$ Steven E. Nissen, ${ }^{6}$ Stuart Pocock, ${ }^{7}$ Neil R. Poulter, ${ }^{8}$ Richard E. Pratley, ${ }^{9}$ Martin Linder, ${ }^{10}$ Tea Monk Fries, ${ }^{10}$ David D. Ørsted, ${ }^{10}$ and Bernard Zinman, ${ }^{11}$ on behalf of the LEADER Trial Investigators*

\title{
OBJECTIVE
}

The Liraglutide Effect and Action in Diabetes: Evaluation of Cardiovascular Outcome Results (LEADER) trial (ClinicalTrials.gov reg. no. NCT01179048) demonstrated a reduced risk of cardiovascular (CV) events for patients with type 2 diabetes who received the glucagon-like peptide 1 receptor agonist liraglutide versus placebo. The mechanisms behind this CV benefit remain unclear. We aimed to identify potential mediators for the CV benefit observed with liraglutide in the LEADER trial.

\section{RESEARCH DESIGN AND METHODS}

We performed exploratory analyses to identify potential mediators of the effect of liraglutide on major adverse CV events (MACE; composite of CV death, nonfatal myocardial infarction, or nonfatal stroke) from the following candidates: glycated hemoglobin $\left(\mathrm{HbA}_{1 \mathrm{c}}\right)$, body weight, urinary albumin-to-creatinine ratio (UACR), confirmed hypoglycemia, sulfonylurea use, insulin use, systolic blood pressure, and LDL cholesterol. These candidates were selected as CV risk factors on which liraglutide had an effect in LEADER such that a reduction in CV risk might result. We used two methods based on a Cox proportional hazards model and the new Vansteelandt method designed to use all available information from the mediator and to control for confounding factors.

\section{RESULTS}

Analyses using the Cox methods and Vansteelandt method indicated potential mediation by $\mathrm{HbA}_{1 \mathrm{c}}$ (up to $41 \%$ and $83 \%$ mediation, respectively) and UACR (up to $29 \%$ and $33 \%$ mediation, respectively) on the effect of liraglutide on MACE. Mediation effects were small for other candidates.

\section{CONCLUSIONS}

These analyses identify $\mathrm{HbA}_{1 \mathrm{c}}$ and, to a lesser extent, UACR as potential mediators of the CV effects of liraglutide. Whether either is a marker of an unmeasured factor or a true mediator remains a key question that invites further investigation.

Liraglutide is a glucagon-like peptide 1 receptor agonist (GLP-1 RA) approved for the management of hyperglycemia in type 2 diabetes and for reduction of cardiovascular (CV) risk in patients with type 2 diabetes and clinical CV disease (CVD) $(1,2)$. It is also approved at a higher dose for the treatment of obesity $(3,4)$. The Liraglutide Effect and Action in Diabetes: Evaluation of Cardiovascular Outcome Results (LEADER) trial was initiated to assess the CV safety of liraglutide in patients with type 2 diabetes and

${ }^{1}$ University of North Carolina School of Medicine, Chapel Hill, NC

${ }^{2}$ Swansea University Medical School, Swansea, U.K.

${ }^{3}$ KfH Kidney Centre, Munich, Germany

${ }^{4}$ Department of Nephrology, Friedrich-Alexander University of Erlangen-Nürnberg, Erlangen, Germany

${ }^{5}$ Diabetes Center Bochum-Hattingen, St. Josef Hospital (Ruhr-Universität Bochum), Bochum, Germany

${ }^{6}$ Cleveland Clinic, Cleveland, $\mathrm{OH}$

${ }^{7}$ Department of Medical Statistics, London School of Hygiene \& Tropical Medicine, London, U.K.

${ }^{8}$ School of Public Health, Imperial College London, London, U.K.

${ }^{9}$ AdventHealth Translational Research Institute, Orlando, FL

${ }^{10}$ Novo Nordisk A/S, Søborg, Denmark

${ }^{11}$ Lunenfeld-Tanenbaum Research Institute, Mount Sinai Hospital, University of Toronto, Toronto, Ontario, Canada

Corresponding author: John B. Buse, jbuse@med .unc.edu

Received 8 November 2019 and accepted 5 April 2020

This article contains supplementary material online at https://doi.org/10.2337/figshare.12106860.

${ }^{*}$ A complete list of the LEADER Trial investigators and members can be found in the supplementary material online. 
showed that it reduced the risk of CV events, all-cause mortality, and renal events compared with placebo $(5,6)$.

Typical CV risk factors associated with type 2 diabetes (glycemic control, body weight, blood pressure, and lipid profiles) are improved by GLP-1 RAs, including liraglutide (7). During the LEADER trial, liraglutide reduced glycated hemoglobin $\left(\mathrm{HbA}_{1 \mathrm{c}}\right)$ and body weight, along with small but significant reductions in systolic blood pressure (6). Liraglutide has also shown multiple direct anti-inflammatory and antiatherosclerotic effects in nonclinical studies $(7,8)$. Given these numerous effects of GLP-1 RAs, it is a challenge to pinpoint the relevant mechanisms underlying the CV benefit of liraglutide $(7,9)$. Mediation analyses allow investigation of the associations among known, measured variables, such as the aforementioned risk factors, and outcomes, but do not necessarily identify causality.

In the present exploratory analyses, we sought to identify potential mediators for the CV benefit observed with liraglutide using data from the LEADER trial. We explored these with several mediation methods, including a new statistical methodology designed to integrate sequential confounders (a limitation of existing methods for mediation analysis) (10).

\section{RESEARCH DESIGN AND METHODS}

\section{Trial Design}

The double-blind, randomized, placebocontrolled LEADER trial assessed the CV safety of liraglutide in the context of standard care in patients with type 2 diabetes, $\mathrm{HbA}_{1 \mathrm{c}} \geq 7 \%$ ( $\geq 53 \mathrm{mmol} / \mathrm{mol}$ ), and a high risk for CVD (aged $\geq 50$ years with established CVD or chronic kidney disease stage 3 or greater or $\geq 60$ years with at least one risk factor for CVD, defined fully in the protocol available as supplementary material to the primary publication [6]). Patients were randomly assigned double-blind, 1:1 to once-daily injections of liraglutide (1.8 mg or maximum tolerated dose) or placebo, both in addition to standard-ofcare treatment for type 2 diabetes and other CV risk factors, with a follow-up period of 3.5-5 years (6).

The primary end point was the time to first occurrence of a major adverse CV event (MACE), including CV death, nonfatal myocardial infarction, or nonfatal stroke (6). Secondary end points included the individual components of the primary composite end point and all-cause death (6). Other variables assessed during the trial included: $\mathrm{HbA}_{1 \mathrm{c}}$, body weight, waist circumference, fasting lipids, systolic blood pressure, diastolic blood pressure, pulse rate, biochemical and hematological parameters, calcitonin levels, anti-liraglutide antibodies, urinary albumin-to-creatinine ratio (UACR), the occurrence of hypoglycemia, adverse events, and concomitant medication use.

\section{Mediation Analyses}

Exploratory analyses were conducted to divide the total effect of liraglutide on time to first MACE, and other time-toevent outcomes, into an indirect effect (also known as mediated effect) and a direct effect (also known as remaining effect). The indirect effect may be interpreted as the difference in time to event between liraglutide and placebo that could be explained by a difference in another variable such as body weight or $\mathrm{HbA}_{1 \mathrm{c}}$, which we will refer to as the mediator. The direct effect is, in contrast, the effect that remained after controlling for the mediator.

Analyses were performed using methods based on a Cox proportional hazards model, which we refer to hereafter as Cox methods, and a new, more complex method, termed the Vansteelandt method (10).

\section{Cox Methods}

Preliminary analyses were performed using two variants of a Cox proportional hazards model to estimate the direct effect:

1. Analysis including the change from baseline in the potential mediator over time as a time-dependent covariate. At any given time point, the most recent change from baseline in the mediator, before the occurrence of the outcome in question, was included as a covariate. Until the first mediator assessment after baseline, the change was 0 . This was one of two approaches used by Inzucchi et al. (11) to evaluate mediated effects on $\mathrm{CV}$ death in the BI 10773 (Empagliflozin) Cardiovascular Outcome Event Trial in Type 2 Diabetes Mellitus Patients (EMPA-REG OUTCOME) trial.

2. Analysis including the updated mean value of the potential mediator (before the occurrence of the outcome in question), calculated as the trapezoidal area under the curve divided by time, as a time-dependent covariate. This was the other approach used by Inzucchi et al. (11) with the intention of capturing the cumulative effect of all prior values of the mediator on the outcome, in contrast to the acute effect in the first approach above.

These two approaches were performed as separate Cox regression analyses for each potential mediator with randomized treatment as a factor and the baseline mediator value as an additional covariate to estimate the direct effect as a log hazard ratio (HR). The total effect was estimated using a model with only treatment as a factor, and the indirect (mediated) effect was estimated as the difference between total and direct effects.

\section{Vansteelandt Method}

The Cox analyses have shortcomings in that they may not capture the full complexity of the mediator and prohibit adequate control for confounding. Furthermore, they do not immediately lend themselves to a counterfactual interpretation (conceiving outcomes in conditions altered from those that were observed) as used in the modern theory of mediation analysis (12). As the main approach for our mediation analysis, we applied a new statistical method proposed by Vansteelandt et al. (10) that addresses these shortcomings and is tailored for time-to-event end points with repeatedly measured mediators.

With this new method, the direct treatment effect was estimated as the difference between probabilities of eventfree survival (i.e., proportions of patients without any event) in liraglutide and placebo groups, instead of an HR. It involved estimation of the counterfactual probability that a patient in the liraglutide group would be event-free at a given time if their mediator values changed to the levels that would have been seen if the patient had been assigned to placebo. Alternatively, this may be interpreted as the survival curve for the liraglutide group after adjusting the mediator values to match those observed in the placebo group. Similar to the Cox method using the updated mean, the Vansteelandt method is based on a dynamic model that incorporates new values of the mediator as they become 
Table 1-Potential mediators of the CV benefit with liraglutide identified from LEADER

\begin{tabular}{lcc} 
Variable & $\begin{array}{c}\text { Difference between liraglutide and } \\
\text { placebo groups }(95 \% \mathrm{Cl})\end{array}$ & $\begin{array}{c}\text { Difference between liraglutide and } \\
\text { placebo groups: } z \text { score }\end{array}$ \\
\hline $\mathrm{HbA}_{1 \mathrm{c}} \%$ & ETD: $-0.40(-0.45 ;-0.34)^{*}$ & -13.5 \\
\hline Body weight, $\mathrm{kg}$ & ETD: $-2.3(-2.5 ;-2.0)^{*}$ & -16.2 \\
\hline UACR & ETR: $0.83(0.78 ; 0.88)^{*}$ & -6.1 \\
\hline Rate of confirmed hypoglycemia during the trial & Rate ratio: $0.81(0.74 ; 0.88)$ & -4.8 \\
\hline Systolic blood pressure, $\mathrm{mmHg}$ & ETD: $-1.2(-1.9 ;-0.5)^{*}$ & -3.3 \\
\hline LDL cholesterol & ETR: $0.98(0.96 ; 0.99)^{*}$ & -3.0 \\
\hline Patients who initiated insulin use during the trial, $n(\%)$ & $1,336(28.8)$ vs. $2,019(43.2)$ & - \\
\hline Patients who initiated SU use during the trial, $n(\%)$ & $349(7.5)$ vs. $505(10.8)$ & - \\
\hline
\end{tabular}

ETD, estimated treatment difference; ETR, estimated treatment ratio; SU, sulfonylurea. ${ }^{*}$ Change from baseline to 36 months (ETR was used when the data were log-transformed, due to their known distributions).

available over time. However, with the Vansteelandt method, mediator values are not summarized into a mean, but are added to the model as separate covariates. The method is therefore more likely to detect a mediated effect if present. All analyses undertaken allowed adjustment for baseline covariates. However, the Vansteelandt method may also adjust for postbaseline confounders, such as concomitant medication, by including them as covariates in the models. Further details of this method have been published separately (10).

The direct effect was estimated as the difference between adjusted survival curve for the liraglutide group (mediator values adjusted to match those in the placebo group) and the actual survival curve for the placebo group. The actual curve was estimated by the same method but using each patient's actual mediator values as covariates.

To estimate the indirect (mediated) effect, the adjusted survival curve for the liraglutide group was compared with the actual curve for the same group. The total effect was estimated by comparing the actual curve for the liraglutide group with the actual curve for the placebo group.

The percentage mediation was calculated as the ratio between the indirect and total effects. Complete mediation would be indicated by a percentage mediation of $100 \%$. The percentage mediation was evaluated and presented at 3 years because this time point corresponds to the last scheduled visit, which all patients should have attended. Thereafter, the number of patients under observation for MACE decreased over time, leading to an increased statistical uncertainty of the estimated survival curves. A Cl for the percentage mediation was estimated using a bootstrap resampling procedure. Because the calculation was highly computer intensive, a $\mathrm{Cl}$ was only calculated for the strongest candidate for mediation.

\section{Identifying Potential Mediators}

Potential mediators examined in all methods included candidate variables on which liraglutide had a clear treatment effect and that could be related to MACE $\left(\mathrm{HbA}_{1 \mathrm{c}}\right.$, body weight, UACR, confirmed hypoglycemic episodes, sulfonylurea use, and insulin use) and candidate mediators on which liraglutide had a modest treatment effect and that could be related to MACE (systolic blood pressure and LDL cholesterol) (Table 1). These previously published treatment effects were evaluated at month $36(6)$, but the mediation analyses undertaken in this study are based on the mediator values indicated in the preceding sections, such as the value at 6 or 12 months, the most recent value, or all values updated over time before the event in question. Each candidate was analyzed separately using the two Cox methods and the Vansteelandt method to explore if it was a potential mediator for MACE.

Additional analyses were performed using the Vansteelandt method for the candidate with the highest apparent degree of mediation as the mediator:

1. Analysis of time to first MACE including the other candidate mediators as confounders.

2. On-treatment analysis of time to first MACE (i.e., an analysis excluding events occurring while the patient was off treatment for $>1$ day). The intention of this analysis was to remove differences in treatment adherence (to either liraglutide or placebo) among patients, because treatment adherence could influence both the mediator and the risk of MACE and thereby act as a confounder. If such confounding existed, the estimated percentage mediation would be expected to be less in the on-treatment analysis than in the main analysis.

3. Analysis of time to first MACE with reversed adjustment for the mediator (i.e., in which the survival curve for the placebo group was estimated after adjusting their mediator values to match those in the liraglutide group). The purpose of this analysis was to explore if the mediator might simply be a marker of exposure to liraglutide. If true, the adjusted survival curve for the placebo group would be expected to be close to the actual placebo curve, indicating no mediation.

4. Analyses of time to all-cause death and $\mathrm{CV}$ death.

\section{RESULTS}

In the LEADER trial, 9,340 patients were randomized to liraglutide $(N=4,668)$ or placebo $(N=4,672)$, in addition to standard-of-care therapy, with a median follow-up of 3.8 years and a median exposure to study drug of 3.5 years (6). To begin this mediation analysis, the published LEADER results were examined (6). Several risk factors were improved in patients in the liraglutide versus placebo groups (Table 1) and hence were analyzed as potential mediators of the previously reported benefit of liraglutide treatment for MACE (HR $0.87[95 \% \mathrm{Cl}$ 0.78 ; 0.97]) (6). 
Cox Methods to Determine Potential Mediators of MACE

Mediation analyses using the Cox methods indicated a mediation effect of $\mathrm{HbA}_{1 \mathrm{c}}$ (Table 2). While there was only a very small mediation effect using the change from baseline as a time-dependent covariate, analysis with the updated mean indicated that $\mathrm{HbA}_{1 \mathrm{c}}$ could be a substantial mediator for the treatment effect of liraglutide on MACE (Table 2). The same analyses with the other potential mediators demonstrated at most a small effect size, with the possible exception for UACR (Table 2).

\section{Vansteelandt Method to Determine Potential Mediators of MACE}

Mediation analysis using the Vansteelandt method (10) also indicated that $\mathrm{HbA}_{1 \mathrm{c}}$ was the most promising candidate among those studied as a potential mediator (Fig. 1 and Table 3). When adjusted for $\mathrm{HbA}_{1 \mathrm{c}}$, the survival curve (proportion of patients without MACE) for patients randomized to liraglutide treatment shifted down toward the placebo curve. This indicated a reduced CV benefit in the liraglutide group when the difference in
$\mathrm{HbA}_{1 \mathrm{c}}$ between the liraglutide and placebo groups was modeled as a mediator for the MACE results (Fig. 1 and Table 3). A potential small mediation effect was observed with UACR (Table 3), but the estimated mediation was relatively small for the other candidates ( $<20 \%$ ) (Table 3 ).

The estimated contribution of $\mathrm{HbA}_{1 \mathrm{c}}$ as a mediator to the effect of liraglutide on MACE at 3 years was $82.0 \%(95 \% \mathrm{Cl}$ $11.7 ; 449.3)$, with the direct effect of liraglutide accounting for the remaining $18 \%$ of the total observed CV benefit (Fig. 1 and Table 3). Because there was no clear mediation through any other candidate variable, none of them would be likely to confound the mediation through $\mathrm{HbA}_{1 \mathrm{c}}$. Nevertheless, the analysis was repeated with body weight, UACR, systolic blood pressure, LDL cholesterol, confirmed hypoglycemic episodes, sulfonylurea use, and insulin use included as confounders in the model. The indirect effect of liraglutide on MACE mediated by $\mathrm{HbA}_{1 \mathrm{c}}$ was estimated to be $69.2 \%$, which is consistent with the analysis without confounders considering the uncertainty in the estimates (Supplementary Fig. 1).
On-treatment analysis (excluding MACE that occurred when a patient was off treatment) showed a slightly stronger mediation effect for $\mathrm{HbA}_{1 \mathrm{c}}$ of $93 \%$ than the $82 \%$ observed in the first Vansteelandt mediation analysis (without confounders) (Supplementary Fig. 2). The reversed adjustment analysis showed a lower but still substantial percentage mediation by $\mathrm{HbA}_{1 \mathrm{c}}$ of $47 \%$ (Supplementary Fig. 3).

\section{Vansteelandt Method Investigating All-Cause Death and CV Death}

The same types of analyses were applied to all-cause death and CV death. The effects of liraglutide on these end points in LEADER appeared to be mediated by $\mathrm{HbA}_{1 \mathrm{c}}$ to some extent (Supplementary Fig. 4).

\section{CONCLUSIONS}

These mediation analyses of the LEADER trial data, with the Cox methods and the new Vansteelandt method, suggest that $\mathrm{HbA}_{1 \mathrm{c}}$ could be a significant mediator of the $\mathrm{CV}$ benefits observed with liraglutide. There was no evidence for a mediation effect with any other candidates that

Table 2-Cox modeling for mediation of time to first MACE with liraglutide versus placebo by $\mathrm{HbA}_{1 \mathrm{c}}$, body weight, UACR, SBP, LDL cholesterol, confirmed hypoglycemic episodes, SU use, and insulin use, assessed using two approaches

\begin{tabular}{|c|c|c|}
\hline Analysis & $\begin{array}{l}\text { Risk of MACE with liraglutide vs. } \\
\text { placebo, } \mathrm{HR}(95 \% \mathrm{Cl})\end{array}$ & $\begin{array}{c}\text { Percentage mediation } \\
(95 \% \mathrm{Cl})^{\dagger}\end{array}$ \\
\hline Total treatment effect (primary LEADER analysis) & $0.868(0.778 ; 0.968)$ & - \\
\hline \multicolumn{3}{|l|}{ Treatment effect adjusted for: } \\
\hline \multicolumn{3}{|l|}{$\mathrm{HbA}_{1 \mathrm{c}}$} \\
\hline Change as time-dependent covariate & $0.880(0.787 ; 0.984)$ & $9.9(-11.4 ; 59.0)$ \\
\hline Updated mean as time-dependent covariate* & $0.920(0.820 ; 1.033)$ & $41.1(8.6 ; 161.0)$ \\
\hline \multicolumn{3}{|l|}{ Body weight } \\
\hline Change as time-dependent covariate & $0.860(0.770 ; 0.962)$ & $-7.4(-43.9 ; 13.3)$ \\
\hline Updated mean as time-dependent covariate* & $0.864(0.772 ; 0.968)$ & $-3.9(-47.0 ; 26.2)$ \\
\hline \multicolumn{3}{|l|}{ UACR } \\
\hline Change as time-dependent covariate & $0.899(0.804 ; 1.004)$ & $21.9(7.5 ; 106.4)$ \\
\hline Updated mean as time-dependent covariate* & $0.907(0.812 ; 1.013)$ & $28.6(12.3 ; 132.8)$ \\
\hline \multicolumn{3}{|l|}{ Confirmed hypoglycemia } \\
\hline Change as time-dependent covariate & $0.868(0.778 ; 0.967)$ & $-0.3(-4.5 ; 2.2)$ \\
\hline Updated mean as time-dependent covariate* & $0.868(0.778 ; 0.968)$ & -0.1 \\
\hline \multicolumn{3}{|l|}{ SBP } \\
\hline Change as time-dependent covariate & $0.874(0.784 ; 0.975)$ & $5.2(-0.6 ; 21.2)$ \\
\hline Updated mean as time-dependent covariate* & $0.877(0.787 ; 0.979)$ & $7.7(0.2 ; 31.7)$ \\
\hline \multicolumn{3}{|l|}{ LDL cholesterol } \\
\hline Change as time-dependent covariate & $0.869(0.778 ; 0.970)$ & $0.6(-4.2 ; 7.7)$ \\
\hline Updated mean as time-dependent covariate* & $0.869(0.778 ; 0.970)$ & $0.7(-4.2 ; 8.8)$ \\
\hline \multicolumn{3}{|l|}{ Insulin use } \\
\hline Change as time-dependent covariate & $0.889(0.796 ; 0.993)$ & $16.9(3.1 ; 85.7)$ \\
\hline Updated mean as time-dependent covariate* & $0.894(0.801 ; 0.998)$ & 20.7 \\
\hline \multicolumn{3}{|l|}{ SU use } \\
\hline Change as time-dependent covariate & $0.861(0.772 ; 0.960)$ & $-5.6(-25.2 ;-0.3)$ \\
\hline Updated mean as time-dependent covariate* & $0.865(0.776 ; 0.965)$ & -2.3 \\
\hline
\end{tabular}

SBP, systolic blood pressure; SU, sulfonylurea. *Calculated as the trapezoidal area under the curve divided by time. $+\mathrm{Cl}$ for the percentage mediation was estimated using a bootstrap resampling procedure. 


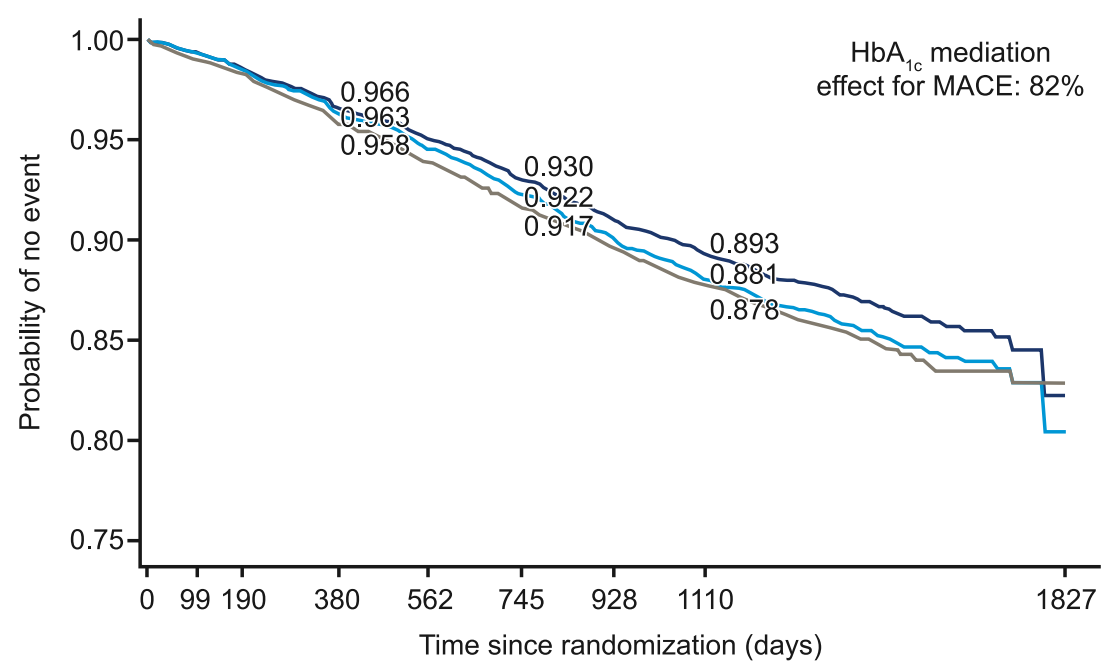

Figure 1-Probability of no MACE occurring in patients treated with liraglutide and placebo and with adjustment for $\mathrm{HbA}_{1 \mathrm{c}}$ as a candidate mediator using the Vansteelandt method.

were assessed in the trial, with the possible exception of UACR. A mediation effect of $\mathrm{HbA}_{1 \mathrm{c}}$ on mortality was also observed. However, it is important to note that these analyses cannot distinguish between the possibilities that $\mathrm{HbA}_{1 \mathrm{c}}$ could be a mediator or a marker of unmeasured factors that are also affected by treatment with liraglutide and that themselves affect $\mathrm{CV}$ outcomes. For example, a large change in $\mathrm{HbA}_{1 \mathrm{c}}$ could be indicative of adherence to treatment, including concomitant medications. Contrary to the hypothesis that $\mathrm{HbA}_{1 \mathrm{c}}$ could be acting as a marker of treatment adherence, an on-treatment analysis showed treatment adherence was unlikely to be a confounding factor, as the percentage mediation was not reduced compared with the main analysis. A related hypothesis is that $\mathrm{HbA}_{1 \mathrm{c}}$ could be acting as a marker of exposure to liraglutide specifically, but this was not supported by the reversed adjustment analysis in which the association between $\mathrm{HbA}_{1 \mathrm{c}}$ and $\mathrm{CV}$ outcomes was also apparent in the placebo group.

Modern CV outcome trials with the GLP-1 RA lixisenatide, dipeptidyl peptidase 4 inhibitors (alogliptin, saxagliptin, and sitagliptin), and insulin (insulin glargine), on a background of standard-ofcare therapy, showed CV safety but failed to show benefits on CV outcomes despite improvements in $\mathrm{HbA}_{1 \mathrm{c}}$ in the active- versus placebo-treatment groups (13-17). More recently, results from other CV outcome trials have shown $\mathrm{CV}$ benefits for the sodium-glucose transporter 2 (SGLT2) inhibitors empagliflozin and canagliflozin and the GLP-1
RAs liraglutide, semaglutide, albiglutide, and dulaglutide $(6,18-22)$. In others, nonsignificant trends for reductions in $\mathrm{CV}$ events (MACE) were reported (for dapagliflozin: SGLT2 inhibitor; for exenatide [once weekly] and semaglutide [oral]: GLP-1 RAs) (23-25). Changes in $\mathrm{HbA}_{1 \mathrm{c}}$ from baseline with the study treatment versus placebo in these more recent trials ranged from $-0.36 \%$ to $-1.0 \%(6,18-25)$, which were marginally larger than changes in the previous trials that showed no $\mathrm{CV}$ benefits (range of changes in $\mathrm{HbA}_{1 \mathrm{c}}$ : $-0.2 \%$ to $-0.36 \%$ [13-17]). These modest changes in $\mathrm{HbA}_{1 \mathrm{c}}$ across all of these trials with varying $\mathrm{CV}$ outcome results (including variation within drug classes) suggest that mechanisms other than reductions in $\mathrm{HbA}_{1 \mathrm{c}}$ account for the $\mathrm{CV}$ benefits with liraglutide, semaglutide, albiglutide, dulaglutide, empagliflozin, and canagliflozin (6,13-25). For example, within Harmony Outcomes, there was a relatively small $(-0.5 \%)$ difference in $\mathrm{HbA}_{1 \mathrm{c}}$ between albiglutide and placebo at 16 months, but a relatively large (22\%) risk reduction in MACE (22).

Epidemiological data support the association between $\mathrm{HbA}_{1 \mathrm{c}}$ and $\mathrm{CV}$ and mortality outcomes $(26,27)$. Data from $>250,000$ people in the Swedish $\mathrm{Na}$ tional Diabetes Register found that $\mathrm{HbA}_{1 \mathrm{c}}$ was a strong predictor of all-cause death, myocardial infarction, stroke, and heart failure (27). These epidemiological data appear to be supported by a metaanalysis using results from early CV outcome trials of intensive glucose control in patients with type 2 diabetes (UK Prospective Diabetes Study [UKPDS], Prospective Pioglitazone Clinical Trial in Macrovascular Events [PROactive], Action in Diabetes and Vascular Disease:

\begin{tabular}{|c|c|c|c|c|}
\hline \multirow[b]{2}{*}{ Variable } & \multicolumn{3}{|c|}{ Estimated proportion of patients without MACE at 3 years } & \multirow[b]{2}{*}{ Percentage mediation $(95 \% \mathrm{Cl})$} \\
\hline & Liraglutide & $\begin{array}{l}\text { Liraglutide, adjusted for } \\
\text { candidate mediator }\end{array}$ & Placebo & \\
\hline $\mathrm{HbA}_{1 \mathrm{c}}$ & 0.893 & 0.881 & 0.878 & $82.0(11.7 ; 449.3)$ \\
\hline Body weight & 0.893 & 0.891 & 0.879 & 14.3 \\
\hline UACR & 0.892 & 0.888 & 0.880 & 33.3 \\
\hline Confirmed hypoglycemia & 0.893 & 0.892 & 0.878 & 6.7 \\
\hline SBP & 0.893 & 0.891 & 0.878 & 13.3 \\
\hline LDL cholesterol & 0.893 & 0.893 & 0.879 & 0.0 \\
\hline Insulin use & 0.892 & 0.890 & 0.878 & 14.3 \\
\hline SU use & 0.893 & 0.891 & 0.878 & 13.3 \\
\hline
\end{tabular}


Preterax and Diamicron MR Controlled Evaluation [ADVANCE], Veterans Affairs Diabetes Trial [VADT], and Action to Control Cardiovascular Risk in Diabetes [ACCORD] - all trials with varying results for CV benefits) showing modest benefits for CV outcomes (28). Taken as a whole, the data strongly suggest that the choice of therapies used to reduce $\mathrm{HbA}_{1 c}$, and not just $\mathrm{HbA}_{1 \mathrm{c}}$ reduction itself, has an important impact on CV outcomes.

For SGLT2 inhibitors, it has been proposed that hemodynamic effectsreducing blood pressure and intravascular volume-and diuretic effects could be underlying mechanisms for the CV benefits $(29,30)$. These hypotheses are supported by a mediation analysis of data from EMPA-REG OUTCOME, which identified hematocrit and hemoglobin, markers of effects on plasma volume, as potential key mediators for the effects of empagliflozin on CV death, with smaller mediation effects attributed to renal function and glycemic control (11). Though $\mathrm{HbA}_{1 \mathrm{c}}$ appears to have a smaller mediation effect relative to hematocrit and hemoglobin for empagliflozin, it was identified as a potential mediator in the EMPA-REG OUTCOME trial based on simple Cox methods (11). The EMPA-REG OUTCOME analyses showed that change from baseline $\mathrm{HbA}_{1 \mathrm{c}}$ mediated $3 \%$ of the effect of empagliflozin on $\mathrm{CV}$ death, while updated mean $\mathrm{HbA}_{1 \mathrm{c}}$ mediated $23 \%$ of the effect, suggesting a greater impact of chronic exposure to elevated $\mathrm{HbA}_{1 \mathrm{c}}$ during the trial (11). Similarly, our analyses of LEADER data using the same two Cox methods (but with the primary end point of time to first MACE) showed little effect of change as a time-dependent covariate, but a larger mediation effect of updated mean $\mathrm{HbA}_{1 \mathrm{c}}$.

The percentage mediation of $\mathrm{HbA}_{1 \mathrm{c}}$ in LEADER is difficult to compare across different analysis methods that we used. However, the Cox analyses appeared to support the main Vansteelandt mediation analyses in identifying $\mathrm{HbA}_{1 \mathrm{c}}$ as the main candidate for a mediator, among those analyzed, of the effect of liraglutide on CV outcomes.

The occurrence of hypoglycemia was shown to be associated with an increased risk of CV events in LEADER (31). However, beyond $\mathrm{HbA}_{1 \mathrm{c}}$ and $\mathrm{UACR}$, little to no mediation effect was identified for the other candidates analyzed, including hypoglycemia. We therefore speculated that factors such as reduction in hypoglycemia combined with a reduction in $\mathrm{HbA}_{1 \mathrm{c}}$ with liraglutide might explain the apparent mediation by $\mathrm{HbA}_{1 \mathrm{c}}$. However, results were largely unchanged when hypoglycemia was included as a confounder (along with other candidates in the mediator model).

Our mediation analyses were post hoc, and, in contrast to the primary analysis of the trial, they are not protected against confounding through patient randomization. The inability to distinguish true mediators from markers is the key limitation of this present and other published mediation analyses. Our analyses are limited by the trial data; only candidate mediators that were examined or measured during the trial could be evaluated. Future trials may help to allow examination of a wider range of potential mediators through collection of biobank samples. However, even in a large trial like LEADER, it was not possible to estimate the percentage mediation with precision. The estimates were subject to substantial uncertainty reflected by the wide $\mathrm{Cl}$ for the percentage mediation through $\mathrm{HbA}_{1 \mathrm{c}}$. It is important to note that there is uncertainty in the total treatment effect of liraglutide in the primary analysis that constitutes the starting point for this mediation analysis and that, based on the $95 \% \mathrm{Cl}$, could be a 3-22\% reduced risk of MACE compared with placebo (6). Furthermore, although the Vansteelandt method has the advantage that it improves the ability to detect a mediated effect, it does not allow us to distinguish what components of the mediator might contribute to the mediated effect (i.e., whether it is the early response, the average level over time within patients, the variability within patients, or a combination of several such components). Also, this analysis was limited to MACE, which is comprised of several individual CVDs, all of which have potentially different underlying pathologies and therefore mediators. These limitations emphasize that the results should be interpreted with caution and only be considered hypothesis generating.

In summary, these mediation analyses have identified $\mathrm{HbA}_{1 \mathrm{c}}$ as a potential mediator of the CV effects of liraglutide. We did not identify any mediation effects for less well-studied but possible candidate mediators, which are also risk factors for CV events, including weight and hypoglycemia. Similar to all other mediation analyses, we cannot necessarily infer causality, and whether $\mathrm{HbA}_{1 \mathrm{c}}$ is a marker of an unmeasured factor or a true mediator remains a key question. Based on existing evidence, we consider it unlikely that $\mathrm{HbA}_{1 \mathrm{c}}$ is a true mediator of the $\mathrm{CV}$ benefit observed with liraglutide, and this finding warrants further investigation.

Acknowledgments. The authors thank all trial personnel and participants and Charlie Hunt and Izabel James of Watermeadow Medical, an Ashfield company (funded by Novo Nordisk), for medical writing and editorial assistance.

Funding. J.B.B. is supported by grants from the National Institutes of Health (UL1TR002489 and P30-DK-124723). J.F.E.M. has received research support from the European Union and Canadian Institutes of Health Research. N.R.P. received grants for his research group relating to type 2 diabetes from Diabetes UK, the National Institute for Health Research Efficacy and Mechanism Evaluation Programme, Julius Clinical, and the British Heart Foundation. R.E.P. has received funding from National Institutes of Health, Foundation for the National Institutes of Health, National Institute on Aging, Winter Park Health Foundation, AdventHealth Orlando, The Elinore and T.W. Miller, Jr. Foundation, Helmsley Charitable Trust, and JDRF. B.Z. has received funding from the National Institutes of Health and Canadian Institutes of Health Research.

Duality of Interest. The LEADER trial (ClinicalTrials.gov identifier NCT01179048) and this post hoc analysis were funded by Novo Nordisk A/S. J.B.B. has received contracted consulting fees paid to the University of North Carolina by Adocia, AstraZeneca, Dance Biopharm Inc., Eli Lilly and Company, MannKind Corporation, NovaTarg Therapeutics, Novo Nordisk, Senseonics, vTv Therapeutics, and Zafgen; receives grant support for clinical trials from Novo Nordisk, Sanofi, and vTv Therapeutics; is a consultant to Cirius Therapeutics, Inc., CSL Behring, Neurimmune AG, and Pendulum Therapeutics; and holds stock options in Mellitus Health, PhaseBio Pharmaceuticals, Inc., Stability Health, and Pendulum Therapeutics. S.C.B. has received research grants (includes principal investigator, collaborator, or consultant and grants) from Health and Care Research Wales (Welsh Government) and Novo Nordisk; has received other research support from Health and Care Research Wales (Welsh Government) and honoraria from Novo Nordisk, Sanofi, Eli Lilly and Company, Boehringer Ingelheim, and Merck; and has an ownership interest in Glycosmedia. J.F.E.M. has received speaker honoraria from AstraZeneca, Boehringer Ingelheim, Eli Lilly and Company, MEDICE, Novo Nordisk, Roche, and Vifor Pharma; has received research support from AstraZeneca, Boehringer Ingelheim, Novo Nordisk, Roche, and Sandoz; and has received consultation fees from AstraZeneca, Bayer, Novo Nordisk, and Vifor Pharma. M.A.N. has served on advisory boards or consulted for AstraZeneca, Boehringer Ingelheim, Eli Lilly and Company, 
Fractyl Laboratories, GlaxoSmithKline, Hanmi Pharmaceutical, Intarcia/Servier, Menarini Group/Berlin Chemie, Merck Sharp \& Dohme, and Novo Nordisk; has received (to his institution) grant support from AstraZeneca, Boehringer Ingelheim, Eli Lilly and Company, GlaxoSmithKline, Intarcia, Menarini Group/Berlin Chemie, Merck Sharp \& Dohme, Novartis Pharmaceuticals, Novo Nordisk A/S, and Sun Pharmaceutical Industries; and has served on the speakers' bureaus of AstraZeneca, Boehringer Ingelheim, Eli Lilly and Company, GlaxoSmithKline, Menarini Group/Berlin Chemie, Merck Sharp \& Dohme, and Novo Nordisk A/S. S.E.N. has received reimbursement from Boehringer Ingelheim and grant support from Pfizer, Amgen Inc., Esperion Therapeutics, CERENIS Therapeutics, The Medicines Company, AstraZeneca, Takeda Pharmaceutical Company, Orexigen Therapeutics, Inc., and Eli Lilly and Company. S.P. received consultancy fees from Novo Nordisk as a member of the LEADER trial Steering Committee. N.R.P. has received speaker fees from Servier, Takeda Pharmaceutical Company, and Novo Nordisk and has served on advisory boards for AstraZeneca and Novo Nordisk. R.E.P. has received research grants from Lexicon Pharmaceuticals Inc., Ligand Pharmaceuticals Incorporated, Eli Lilly and Company, Merck, Novo Nordisk, Sanofi US LLC, and Takeda Pharmaceutical Company; has acted as a speaker for AstraZeneca, Novo Nordisk, and Takeda Pharmaceutical Company; and has acted as a consultant for Glytec LLC, Mundipharma, Sanofi, AstraZeneca, Boehringer Ingelheim, Eisai Inc., GlaxoSmithKline, Janssen Scientific Affairs, LLC, LigandPharmaceuticals Incorporated, Eli Lilly and Company, Merck, Novo Nordisk, Pfizer, and Takeda Pharmaceutical Company (all payments made directly to employer AdventHealth Translational Research Institute). M.L. is a full-time employee of Novo Nordisk. T.M.F. and D.D. $\varnothing$. are full-time employees of and hold stock in Novo Nordisk. B.Z. has received consulting fees from Merck, Novo Nordisk, Sanofi, Eli Lilly and Company, AstraZeneca, Janssen, and Boehringer Ingelheim. No other potential conflicts of interest relevant to this article were reported.

Author Contributions. J.B.B., J.F.E.M., M.A.N., S.E.N., S.P., N.R.P., and B.Z. were members of the LEADER trial steering committee. J.B.B., J.F.E.M., M.A.N., S.E.N., N.R.P., and B.Z. are current members of the LEADER publication committee, and all contributed significantly to the design and conduct of the study and acquisition of clinical data. S.C.B. and R.E.P. are members of the LEADER publication committee, were trial investigators, co-chaired the global expert panel, and contributed to conduct of the study and acquisition of clinical data. M.L. performed the statistical analyses. All authors reviewed and interpreted the data and were involved in drafting and critical revision of the manuscript. All authors approved the final version of the manuscript and take full responsibility for the content. J.B.B. is the guarantor of this work and, as such, had full access to all of the data in the study and takes responsibility for the integrity of the data and the accuracy of the data analysis.

\section{References}

1. Novo NordiskA/S. Victoza (liraglutide)-Summary of Product Characteristics [Internet], 2019.
Available from https://www.ema.europa.eu/en/ documents/product-information/victoza-eparproduct-information_en.pdf. Accessed 23 April 2020

2. Novo Nordisk A/S. Victoza (liraglutide) - Highlights of prescribing information [Internet], 2019. Available from https://www.accessdata.fda.gov/ drugsatfda_docs/label/2019/022341s031lbl.pdf. Accessed 23 April 2020

3. Novo Nordisk A/S. Saxenda (liraglutide) Summary of Product Characteristics [Internet] 2019. Available from https://www.ema.europa .eu/en/documents/product-information/saxendaepar-product-information_en.pdf. Accessed 23 April 2020

4. Novo Nordisk A/S. Saxenda (liraglutide) Highlights of prescribing information [Internet], 2018. Available from https://www.accessdata.fda .gov/drugsatfda_docs/label/2018/206321s007lbl .pdf. Accessed 23 April 2020

5. Mann JFE, Ørsted DD, Brown-Frandsen K et al.; LEADER Steering Committee and Investigators. Liraglutide and renal outcomes in type 2 diabetes. N Engl J Med 2017;377:839-848

6. Marso SP, Daniels GH, Brown-Frandsen K, et al.; LEADER Steering Committee; LEADER Trial Investigators. Liraglutide and cardiovascular outcomes in type 2 diabetes. N Engl J Med 2016;375: 311-322

7. Drucker DJ. The cardiovascular biology of glucagon-like peptide-1. Cell Metab 2016;24: 15-30

8. Rakipovski G, Rolin B, Nøhr J, et al. The GLP-1 analogs liraglutide and semaglutide reduce atherosclerosis in $\mathrm{ApoE}^{-/-}$and $\mathrm{LDLr}^{-/-}$mice by a mechanism that includes inflammatory pathways. JACC Basic Transl Sci 2018;3:844-857

9. Nauck MA, Meier JJ, Cavender MA, Abd El Aziz $M$, Drucker DJ. Cardiovascular actions and clinical outcomes with glucagon-like peptide-1 receptor agonists and dipeptidyl peptidase-4 inhibitors. Circulation 2017;136:849-870

10. Vansteelandt $S$, Linder $M$, Vandenberghe $S$ Steen J, Madsen J. Mediation analysis of timeto-event endpoints accounting for repeatedly measured mediators subject to time-varying confounding. Stat Med 2019;38:4828-4840

11. Inzucchi SE, Zinman B, Fitchett D, et al. How does empagliflozin reduce cardiovascular mortality? Insights from a mediation analysis of the EMPA-REG OUTCOME trial. Diabetes Care 2018; 41:356-363

12. VanderWeele TJ. Explanation in Causal Inference: Methods for Mediation and Interaction. New York, Oxford University Press, 2015

13. Green JB, Bethel MA, Armstrong PW, et al.; TECOS Study Group. Effect of sitagliptin on cardiovascular outcomes in type 2 diabetes. N Engl J Med 2015;373:232-242

14. Gerstein HC, Bosch J, Dagenais GR, et al.; ORIGIN Trial Investigators. Basal insulin and cardiovascular and other outcomes in dysglycemia. N Engl J Med 2012;367:319-328

15. Pfeffer MA, Claggett B, Diaz R, et al.; ELIXA Investigators. Lixisenatide in patients with type 2 diabetes and acute coronary syndrome. N Engl J Med 2015;373:2247-2257

16. Scirica BM, Bhatt DL, Braunwald E, et al.; SAVOR-TIMI 53 Steering Committee and Investigators. Saxagliptin and cardiovascular outcomes in patients with type 2 diabetes mellitus. N Engl J Med 2013;369:1317-1326
17. White $W B$, Cannon $C P$, Heller $S R$, et al.; EXAMINE Investigators. Alogliptin after acute coronary syndrome in patients with type 2 diabetes. N Engl J Med 2013;369:1327-1335

18. Gerstein HC, Colhoun HM, Dagenais GR, et al.; REWIND Investigators. Dulaglutide and cardiovascular outcomes in type 2 diabetes (REWIND): a double-blind, randomised placebocontrolled trial. Lancet 2019;394:121-130

19. Marso SP, Bain SC, Consoli A, et al.; SUSTAIN6 Investigators. Semaglutide and cardiovascular outcomes in patients with type 2 diabetes. N Eng J Med 2016;375:1834-1844

20. Neal B, Perkovic V, Mahaffey KW, et al. CANVAS Program Collaborative Group. Canagliflozin and cardiovascular and renal events in type 2 diabetes. N Engl J Med 2017;377:644657

21. Zinman B, Wanner C, Lachin JM, et al.; EMPAREG OUTCOME Investigators. Empagliflozin, cardiovascular outcomes, and mortality in type 2 diabetes. N Engl J Med 2015;373:2117-2128

22. Hernandez AF, Green JB, Janmohamed S, et al.; Harmony Outcomes committees and investigators. Albiglutide and cardiovascular outcomes in patients with type 2 diabetes and cardiovascular disease (Harmony Outcomes): a double-blind, randomised placebo-controlled trial. Lancet 2018;392:1519-1529

23. Holman RR, Bethel MA, Mentz RJ, et al.; EXSCEL Study Group. Effects of once-weekly exenatide on cardiovascular outcomes in type 2 diabetes. N Engl J Med 2017;377:1228-1239

24. Husain M, Birkenfeld AL, Donsmark M, et al.; PIONEER 6 Investigators. Oral semaglutide and cardiovascular outcomes in patients with type 2 diabetes. N Engl J Med 2019;381: 841-851

25. Wiviott SD, Raz I, Bonaca MP, et al.; DECLARE-TIMI 58 Investigators. Dapagliflozin and cardiovascular outcomes in type 2 diabetes. $\mathrm{N}$ Engl J Med 2019;380:347-357

26. Khaw KT, Wareham N, Bingham S, Luben $R$, Welch A, Day N. Association of hemoglobin A1c with cardiovascular disease and mortality in adults: the European Prospective Investigation into Cancer in Norfolk. Ann Intern Med 2004;141: 413-420

27. Rawshani A, Rawshani A, Franzén $S$, et al. Risk factors, mortality, and cardiovascular outcomes in patients with type 2 diabetes. N Engl J Med 2018;379:633-644

28. Ray KK, Seshasai SR, Wijesuriya S, et al. Effect of intensive control of glucose on cardiovascular outcomes and death in patients with diabetes mellitus: a meta-analysis of randomised controlled trials. Lancet 2009;373:1765-1772

29. Sattar N, McLaren J, Kristensen SL, Preiss D, McMurray JJ. SGLT2 Inhibition and cardiovascular events: why did EMPA-REG Outcomes surprise and what were the likely mechanisms? Diabetologia 2016;59:1333-1339

30. Staels B. Cardiovascular protection by sodium glucose cotransporter 2 inhibitors: potential mechanisms. Am J Cardiol 2017;120: S28-S36

31. Zinman B, Marso SP, Christiansen E, Calanna $\mathrm{S}$, Rasmussen S, Buse JB; LEADER Publication Committee on behalf of the LEADER Trial Investigators. Hypoglycemia, cardiovascular outcomes, and death: the LEADER experience. Diabetes Care 2018;41:1783-1791 